\title{
Editorial
}

\section{Is Virtual Simulation a Reality?}

\author{
J. Scott Ferguson \\ Division of Pulmonary and Critical Care Medicine, University of Wisconsin, Madison, Wisc., USA
}

On July 1, 1994, I reported for fellowship training and was informed that I would be on the bronchoscopy service. My attending showed me how to perform a bronchoscopy. Procedure numbers 2 through 7 that day were done with me bumbling my way down the airway, no doubt causing certain anxiety if not discomfort to my patients.

As excited as I was that first day, nearly 16 years later I no longer consider 'learning by doing' to be appropriate training in bronchoscopy skills for new learners. Many pulmonologists and other practitioners of the art of bronchoscopy agree and are interested in proactive teaching models within realistic environments without the risk of harm to patients. Although recent work [1] has shed light on methods to improve the age old model of 'see one, do one, teach one,' serious questions remain concerning the number of procedures needed to be proficient, the number needed to remain proficient once trained, the most appropriate model to use, and how to reliably measure learning in this procedure.

In this issue of Respiration, Konge et al. [2] add important knowledge to a growing body of work regarding assessment of bronchoscopy skills. Using virtual reality simulation with 6 cases that increased in difficulty, they tested novice, in-training, and expert bronchoscopists and used a check list metric that was designed to reduce subjectivity. One unblinded rater judged each procedure, and 2 blinded raters judged a portion of these by use of video recorded procedures. The checklist allowed gen-

\section{KARGER}

Fax +4161306 1234

E-Mail karger@karger.ch

www.karger.com
(C) 2011 S. Karger AG, Basel

0025-7931/11/0816-0446\$38.00/0

Accessible online at:

www.karger.com/res eration of a bronchoscopy score that was then divided by the time of the procedure to generate an efficiency score. Also tested was the ability of the built-in simulator metrics to distinguish bronchoscopists' skills.

Importantly, the metric as used was able to discriminate 3 groups of bronchoscopists with a priori divergent skill levels. Significant differences among the 3 levels of bronchoscopists were found for the bronchoscopy score, the time of the procedure, and the efficiency score. Interestingly, the built-in metrics included on the simulator were not able to distinguish the 3 bronchoscopy skill levels, except for the time of the procedure.

These results are important for several reasons. The authors have demonstrated the ability to quantify the difference in skill level among 3 levels of skill in a virtual simulator. This is not only important for initial training but may also be important if virtual reality is used in the maintenance of skills. Additionally, this is a method that reduces subjectivity in the assessment. While some subjective measures (e.g. comfort level, posture, and interaction with the patient) are important and should not be excluded from assessment, strong objective measures are desirable to improve the standardization of testing across a variety of institutions. Also, using a virtual reality environment will conceivably have fewer variations in standardization and is likely to lend itself well to the creation of a variety of clinically applicable scenarios that may be modified to allow for adding new skills or refreshment of old ones. 
While the current study adds new information and creates a tool that is likely of value to our training programs in bronchoscopy, many questions remain. We do not yet know if the virtual reality environment is the right model for training endoscopy skills or whether training can in part be done with lower cost mannequin models. While very close to reality, virtual simulation of complications, emergencies, and advanced skills is more difficult than virtual simulation of airway exams and biopsies, but it is likely to be important for continued education. If instructors implement advanced virtual simulation in their programs, it is not clear how multiple programs can afford these high-cost items or if efforts should be focused on cost-sharing arrangements and further development of low-fidelity models. Most importantly, at this time it is not clear how training on models, virtual or otherwise, translates into quality patient care.
These and many more questions will be asked and answered in future studies. No matter what the answers, as educators and physicians we owe it to our patients to continue to strive for the best education for our students. As a possible future patient of my current students, I hope that virtual simulation becomes reality.

References

1 Davoudi M, Osann K, Colt HG. Validation of two instruments to assess technical bronchoscopic skill using virtual reality simulation. Respiration 2008;76:92-101.

- 2 Konge L, Arendrup H, von Buchwald C, Ringsted C: Using performance in multiple simulated scenarios to assess bronchoscopy skills. Respiration 2011;81:483-490. 